\title{
Establishment And Validation Of Coagulation Factor-based Nomogram For Predicting The Recurrence-free Survival Of Prostate Cancer
}

\section{Jialin Meng}

Anhui Medical University

\section{Zichen Bian}

Anhui Medical University

Chenyu Zhu

Anhui Medical University

\section{Zhi Tao}

Anhui Medical University

\section{Xiaoyan Jin}

Anhui Medical University

Jun Zhou

Anhui Medical University

\section{Meng Zhang}

Anhui Medical University

Chaozhao Liang ( $\sim$ liang_chaozhao@ahmu.edu.cn )

Department of Urology, The First Affiliated Hospital of Anhui Medical University and Institute of Urology and Anhui Province Key Laboratory of Genitourinary Diseases, Anhui Medical University, Jixi Road 218th, Shushan District, Hefei, Anhui, 230022, People's Republic of China https://orcid.org/0000-0003-23171323

\section{Research article}

Keywords: Prostate cancer, recurrence-free survival, coagulation pathway, Nomogram

Posted Date: January 14th, 2021

DOI: https://doi.org/10.21203/rs.3.rs-143707/v1

License: (c) (i) This work is licensed under a Creative Commons Attribution 4.0 International License. Read Full License 


\section{Abstract}

Background: We aimed to establish and validate a coagulation-feature-based nomogram to predict recurrence-free survival for prostate cancer patients.

Methods: The study contained 168 prostate cancer patients who had received radical prostatectomy between 2012 and 2018. The Kaplan-Meier plot and log-rank analysis were used to screen recurrence-free survival-related features. The nomogram was established by combining the significant coagulation features with clinicopathological characteristics by using Cox regression analysis. The accuracy and clinical significance of the nomogram model were assessed by receiver operating characteristic (ROC) curve, Kaplan-Meier plot, and calibration plot. Besides, we explored the correlation between coagulation pathway activity and patients' prognosis based on public datasets by using gene set variation analysis (GSVA) analysis.

Results: The results suggested that patients in the high-risk subgroup showed unfavorable prognoses than those in the low-risk subgroup classified by the nomogram model in both the training (log-rank $P<$ 0.0001 ) and validation (log-rank $P=0.0004$ ) cohorts. The nomogram model exhibited high discriminative accuracy in the training cohort [1-year area under the curve (AUC) of 0.74, and 3-year AUC of 0.69], which was confirmed in the internal validation cohort $(C$-index $=0.651)$. Besides, the calibration plots confirmed good concordance for the prediction of recurrence-free survival at 1 and 3 years. Besides, the subgroup analyses confirmed the usage of this model in different clinicopathological subgroups. Finally, GSVA analyses suggested that patients with higher coagulation pathway scores mostly had unfavorable prognoses than those with lower scores, a result consistent with the findings obtained above.

Conclusions: In conclusion, we develop a practical nomogram model for the recurrence-free survival predicting of prostate cancer patients. This model may offer clinicians prognostic assessments and facilitate personalized treatment.

\section{Highlights}

1. It becomes urgent to establish a suitable and feasible prognostic model to guide clinical treatment;

2. A coagulation factor-based nomogram effectively predicts the recurrence-free survival prognosis of prostate cancer;

3. Activation of the coagulation pathway is associated with an unfavorable prognosis of prostate cancer

\section{Background}

Prostate cancer is positively related to the male's reproductive system, and take the second high incidence of cancer in men [1]. In Asian countries, there are 3.8 per 100,000 people died from prostate cancer each year, which causes severe health and economic problems to society [2]. Male dead from 
prostate cancer usually had a close relationship with metastasis and castration-resistance [3]. Though the survival was improved with the development of therapeutic strategies, the heterogeneous biological processes of prostate cancer in different patients lead to diverse clinical outcomes [3-5].

Recently, the predicted value of clinical parameters in different tumors has been focused and numerous publications emerged [6-9], such as prostate cancer [10, 11]. In recent years, the association between coagulation disorders and tumorigenesis and tumor progression has attracted the attention of many researchers. On the one hand, malignancy always along with a hypercoagulable state, the presence of tissue factor and thrombin leads to the coagulation of cancer patients and about $15 \%$ of the patients will face the episode of thrombotic [12-14]. On the other hand, the thrombin will impact the angiogenesis of the tumor through releasing the anti-vascular endothelial growth factor (VEGF) by the platelet a granules, it can also promote the proliferation and migration of endothelial cells in the condition of tissue damage $[15,16]$. Lin et al.[17] reported that plasma D-dimer and fibrinogen were robust predictors of poor survival in digestive cancer patients. Cao et al. revealed that the fibrinogen/albumin ratio index is an independent prognosis predictor of recurrence-free survival in patients after surgical resection of gastrointestinal stromal tumors. However, the prognostic value of coagulation factors in prostate cancer patients has not been mentioned.

In the current study, we enrolled all the clinical laboratory results of coagulation factors of 168 prostate cancer patients, as well as the essential clinicopathological features, along with the follow-up record. Nomogram was employed to illustrate the new findings of the predicted value of coagulation factors for the recurrence-free survival outcome.

\section{Methods}

\section{Study population}

Patients diagnosed with prostate cancer by the prostate biopsy in The Department of Urology, The First Affiliated Hospital of Anhui Medical University from 2012 to 2018 were involved in the study. They should also meet the following criteria: (1) with the results of coagulation factors including prothrombin time (PT), international normalized ratio (INR), plasma thromboplastin antecedent (PTA), activated partial thromboplastin time (APTT), fibrinogen (FIB), thrombin time (TT), fibrin degradation products (FDP), Ddimer (DD); (2) with the record of age, Gleason score, pathological T stage, and total prostate-specific antigen (TPSA) value; 3 ) the laboratory tests should be one the same instrument and with same reference range; otherwise, patients who suffered from hematological diseases or took anticoagulant medicine were excluded. Patients were regularly followed up by telephone, mail, or in the clinic, and the endpoint of the primary outcome was the biochemical recurrence, which was defined with the presence of the TPSA level greater than $0.2 \mathrm{ng} / \mathrm{mL}$ after radical prostatectomy, followed by a confirmatory test showing a persistent TPSA level greater than $0.2 \mathrm{ng} / \mathrm{mL}$ [1].

Public databases and gene set variation analysis (GSVA) 
To evaluate the function and prognostic value of coagulation, we analyzed four public databases, The Cancer Genome Atlas Prostate Adenocarcinoma (TCGA-PRAD) cohort, Memorial Sloan Kettering Cancer Center (MSKCC) cohort, GSE116918 cohort, GSE70769 cohort. We acquire the RNA-seq data from a public data hub, UCSC Xena (https://xenabrowser.net/) for the TCGA-PRAD, including 495 prostate cancer samples. For the other three cohorts, the gene expression profiling by array was downloaded from GEO (https://www.ncbi.nlm.nih.gov/geo). Then the coagulation pathways activated score was generated by the Gene Set Variation Analysis (GSVA) analysis with the estimation variation of the enrichment of the genes in HALLMARK_COAGULATION_PATHWAY

(https://www.gseamsigdb.org/gsea/msigdb/cards/HALLMARK_COAGULATION). As for the assessment of the recurrent-free survival of patients with coagulation pathways active status, the K-M survival analysis was performed.

\section{Statistical analyses}

All the patients were randomly separated into the training cohort and validation cohort with a ratio of 7:3. The cut-off value for age was set as 60 years old ( $>60 \mathrm{vs}<=60$ ); for PSA was $10 \mathrm{ng} / \mathrm{ml}(>10 \mathrm{vs} .<=10$ ), and the Gleason Group was used to divide the different stages of PCa; otherwise, the cut-off value of coagulation factors was determined by the median value. The gene set enrichment score was conducted by the "GSVA" R package. Nomogram was performed by the "RMS" package to establish the predicted signature by the time and status dependent Cox regression analysis. K-M survival analysis was used to evaluate and select the prognostic coagulation factors with the "survminer" R package, as well as to show the predictive value of the Nomogram. The accuracy of the nomogram for predicting 1-year and the 3year recurrent rate was evaluated by calibration curve by "RMS" package (bootstraps with 1000 resample), while Hosmer-Lemeshow test was applied to detect the goodness of fit for the Nomogram. Moreover, the predictability of both the established Nomogram and the generated points was assessed by the receiver operating characteristic (ROC) curve with the "ROCR" package. R software was used for all statistical analyses and the statistical significance was decided on the two-tailed $P<0.05$.

\section{Results}

\section{The prognostic value of coagulation factors}

Fig. 1 showed the design of current study. The K-M survival analysis was used to judge the recurrent-free survival of patients with different coagulation function status. The median value of these coagulation factors used to separate the patients to high-level and low-level subgroups. We found that the lower value of FDP (HR: $0.55,95 \% \mathrm{Cl}: 0.314-0.951, P=0.033$ ), and TT (HR: $0.54,95 \% \mathrm{Cl}: 0.314-0.920, P=0.024$ ) are the risk factor of the biochemical recurrence of prostate cancer patients (Fig. 2). While the other factors, PT (HR: 1.04, 95\% Cl: 0.611-1.768, $P=0.89$ ), PTA (HR: 1.01, 95\% Cl: 0.585-1.751, $P=0.965)$, FIB (HR: 0.7, 95\% Cl: 0.404-1.205, $P=0.197$ ), INR (HR: $1.08,95 \% \mathrm{Cl}: 0.629-1.855, P=0.78)$, APTT (HR: $1.43,95 \% \mathrm{Cl}$ : $0.839-2.441, P=0.189)$ and DD (HR: $0.84,95 \% \mathrm{Cl}: 0.493-1.446, P=0.537)$ are not the predicative factors 
for the biochemical recurrence for prostate cancer patients. Thus, we considered containing TT and FDP into our nomogram model.

\section{Separation of the training and validation cohorts}

With the ratio of 7:3, prostate cancer patients were separated into the training $(n=120)$ and validation sets $(n=48)$ randomly, and the patients' characteristics in both cohorts were summarized in Table 1 . The distribution of patients with different clinical features consistent among the training and validation cohorts (all $P>0.05$ ). The recurrent-free survival time in the training cohort is $19.66 \pm 16.98$ months, while the recurrent-free survival time in the validation cohort is $19.66 \pm 15.37$ months.

Establish and validate the prognostic nomogram for prostate cancer patients in the training cohort

Other than the TT and FDP parameters, we also enrolled the clinical features of age, TPSA, and Gleason Group, which are traditional used for the predicting of the severe status of prostate cancer patients in clinical, to establish the prognostic nomogram (Fig.3). We also tried to enrolled the pathological T stage to establish the nomogram model, but the overall prediction value was less good than without it (data not shown). Patients can obtain the total points along with the value of age, TPSA, Gleason Group, TT, and FDP refer to the nomogram model, and the specific points for each value listed in Table 2 . We observe that younger age is a risk factor for the recurrence of prostate cancer patients, while no doubt results that higher TPSA and advanced Gleason Group is the risk factor. Referring to the median risk score, we subclassified the prostate cancer patients into high- and low-risk of recurrence in the training cohort (highrisk group vs. low-risk group: recurrent-free survival time $25.49 \pm 19.45$ months vs. $14.11 \pm 12.23$ months, log-rank $P$-value $<0.0001$, Fig. 4A). The stability and predictive value were also proved by calibration plot

(Fig. 4B) and C-index analyses (C-index $=0.74$ for 1-year and 0.69 for 3-year of recurrent-free survival prediction, Fig. 4C).

\section{ROC and K-M analyses for prostate cancer patients in the validation cohort}

We calculated the total points of patients in the validation cohort according to the well-established Nomogram model based on the training cohort. K-M plot and log-rank analysis proved that the patients in the high-risk subgroup had unfavorable prognoses than the low-risk subgroup (high-risk group vs. low-risk group: recurrent-free survival time $25.31 \pm 16.50$ months vs. $14.67 \pm 12.93$ months, log-rank $P$-value $=$ 0.004 , Fig. $5 \mathrm{~A}$ ). The predictive value of the Nomogram was confirmed by ROC analyses again in the validation cohort, with the AUC value of 0.651 (Fig. 5B).

To verify whether this nomogram model could be used in different clinicopathological subgroups, we performed subgroup analyses. We found that this model was able to predict the recurrence-free survival of prostate cancer patients in different age subgroups ( $<=60$, and $>60$ years old) and PSA level subgroups ( $<=10 \mathrm{ng} / \mathrm{ml}$, and $>10 \mathrm{ng} / \mathrm{ml}$ ) (Fig. 6). Attributing to the limited samples in the "Gleason score $<7$ " subgroup, we failed to obtain positive results (Fig. 6). Future studies are needed to verify this finding. 
As mentioned above, the activation of the coagulation system might play important roles during the progression of prostate cancer. We thought to explore the relationship between coagulation function status and recurrent-free survival of prostate cancer patients at genetic levels. We acquired the mRNA expression profile of prostate cancer samples from four public datasets [TCGA-PRAD cohort (495 samples), MSKCC cohort (140 samples), GSE116918 cohort (248 samples), and GSE70769 cohort (93 samples)]. Then, the coagulation pathway activated score for each patient from the online website GSVA was calculated. Our results suggested that these patients with higher coagulation scores mostly had unfavorable prognoses than those with lower coagulation scores in the MSKCC cohort $(P=0.023)$ and GSE116918 ( $P=0.012)$ (Fig. 7). We failed to obtain positive results for the other two cohorts, even the curves showed good tendency (TCGA-PRAD cohort, $P=0.082$, and GSE70769 cohort, $P=0.261$, Fig. 7).

\section{Discussion}

Although significant improvements have been achieved in treating prostate cancer, recurrence cannot be avoided. Necessary efforts should be poured into the searching of dependable prognostic biomarkers to help the individualized treatment for prostate cancer patients. Notably, the tumor procession is usually presented with several symbols like the promotion of metastasis, invasion, and development, whereas, these activities of the tumor has already been shown to have a close relationship with hemostatic activities [18-20].

We employed K-M survival analysis and log-rank analysis and found that the lower value of FDP (HR: $0.55,95 \% \mathrm{Cl}: 0.31-0.95, P=0.033)$, and TT (HR: 0.54, 95\% Cl: $0.314-0.920, P=0.024$ ) are the risk factor of the biochemical recurrence of prostate cancer patients (Fig. 1). Thus, we considered containing TT and FDP into our nomogram model. Other than these two parameters, we also enrolled the clinical features of age, TPSA, and Gleason score, which are traditionally used for the predicting of the severe status of prostate cancer patients in clinical, to establish the prognostic nomogram. The predictive value of the nomogram was proved by K-M plot, ROC, DCA, calibration analyses in both the training and validation cohorts. Besides, since activation of the coagulation system might play important roles during the progression of prostate cancer, we thought to explore the relationship between coagulation function status and recurrent-free survival of prostate cancer patients at genetic levels. Our results suggested that these patients with higher coagulation scores mostly had unfavorable prognoses than those with lower coagulation scores in the MSKCC cohort $(P=0.023)$ and GSE116918 $(P=0.012)$. Our results highlight the prognostic role of coagulation markers.

Clinically, it is feasible and inexpensive to test the levels of FDP and TT in clinical laboratories, which could reflect the function of coagulation, anticoagulation, and fibrinolysis. Thrombin is produced by inactive prothrombin precursors, which contributes to coagulation activation after being digested by factor Xa. It then acts as a catalyst to catalyze the crosslinking of insoluble fibrin to form soluble fibrinogen. The plasmin-mediated degradation of fibrinogen or fibrin releases fragments and formats Fibrinogen (fibrin) [21]. Research done by Li et al. [22] proved that the reduction in pretreatment TT has a negative impact on the overall survival of esophageal carcinoma (ESCC) while a study finished by 
Mischke et al. [23] found that a low level of FDP was able to lead to the reduce of TT. These researches remind us that FDP may influence the level of TT in prostate cancer patients. A short time of TT suggested the coagulation system was activated, and the results were consistent with the GSVA pathway analysis that higher activation of the coagulation pathway is associated with unfavorable prognosis of prostate cancer patients.

Several advantages and limitations should be noted here. Currently, although many prognostic models have been established and some of them are already been used clinically, few of them are easily accessed, and entirely foolproof. Molecular events reveal different biological behaviors based on various clinics, which may help to make risk-stratified clinical decisions and personal prognosis prediction [24, 25]. For the limitation aspect, the sample size of our study was not full enough, and the results needed to be validated externally. Besides, our results were based on continuous variables. If future studies want to extend current findings, the values of TT, PSA, and FDP should be obtained from the same type of instrument and with the same reference range.

\section{Conclusion}

Our study establishes a nomogram model for prostate cancer patients based on easily accessible metrics from clinicopathological and laboratory tests. These findings are warranted to be validated by multicenter prospective studies.

\section{List Of Abbreviations}

Receiver operating characteristic, ROC; Gene set variation analysis, GSVA; Prothrombin time, PT; International normalized ratio, INR; Plasma thromboplastin antecedent, PTA; Activated partial thromboplastin time, APTT; Fibrinogen, FIB; Thrombin time, TT; Fibrin degradation products, FDP; Ddimer, DD; Total prostate-specific antigen, TPSA; The Cancer Genome Atlas Prostate Adenocarcinoma, TCGA-PRAD; Memorial Sloan Kettering Cancer Center, MSKCC;

\section{Declarations}

\section{Ethics approval and consent to participate}

The research contents and research programs were reviewed and approved by the Ethics Committee of the First Affiliated Hospital of Anhui Medical University (anyiyifuyuanlunshen-kuai-PJ-2019-09-11). As this study is a retrospective study, only limited data which is known to the researchers was colletcted and the process of the study will not affect treatment or the patients' clinical care and the patients have left the hospital already. Therefore, the consent to review the medical records were not required by the Ethics Committee of the First Affiliated Hospital of Anhui Medical University. Meanwhile, the patients has been orally informed that their medical records may be used to make clinical researchs before they accept the operation. 


\section{Consent for publication}

All authors have read and approved the manuscript being submitted and published, and agree to its submitted to this journal.

\section{Availability of data and materials}

The anonymized data used and analysed during the current study are available from the corresponding authors on reasonable request.

\section{Competing interests}

The authors declare that they have no competing interests.

\section{Funding}

Chaozhao Liang funded the study with the National Natural Science Foundation of China (\#81802827 and \#81630019) and Scientific Research Foundation of the Institute for Translational Medicine of Anhui Province (\#2017ZHYX02). Meng Zhang funded the study with The Natural Science Foundation of Guangdong Province, China (\#2017A030313800).

\section{Authors contributions}

Conception and Design: $\mathrm{CL}, \mathrm{JM}$, and MZ. Collection and Assembly of Data: $\mathrm{XJ}, \mathrm{CZ}, \mathrm{ZB}, \mathrm{ZT}$. Data Analysis and Interpretation: MZ. Manuscript Writing: JM, MZ, ZB. Final Approval of Manuscript: All the authors.

\section{Acknowledgments}

The authors wish to thank the Center for Scientific Research of the First Affiliated Hospital of Anhui Medical University for valuable help in our experiments.

\section{References}

1. Khazaei Z, Sohrabivafa M, Momenabadi V, Moayed L, Goodarzi E: Global cancer statistics 2018: Globocan estimates of incidence and mortality worldwide prostate cancers and their relationship with the human development index. 2019, 9(3):245-250.

2. Hassanipour S, Delam H, Arab-Zozani M, Abdzadeh E, Hosseini SA, Nikbakht HA, Malakoutikhah M, Ashoobi MT, Fathalipour M, Salehiniya $\mathrm{H}$ et al: Survival Rate of Prostate Cancer in Asian Countries: A Systematic Review and Meta-Analysis. Annals of global health 2020, 86(1):2.

3. Beer TM, Armstrong AJ, Rathkopf DE, Loriot Y, Sternberg CN, Higano CS, Iversen P, Bhattacharya S, Carles $\mathrm{J}$, Chowdhury $\mathrm{S}$ et al: Enzalutamide in metastatic prostate cancer before chemotherapy. $N$ Engl J Med 2014, 371(5):424-433. 
4. Scher HI, Fizazi K, Saad F, Taplin ME, Sternberg CN, Miller K, de Wit R, Mulders P, Chi KN, Shore ND et al: Increased survival with enzalutamide in prostate cancer after chemotherapy. The New England journal of medicine 2012, 367(13):1187-1197.

5. Gillessen S, Attard G, Beer TM, Beltran H, Bossi A, Bristow R, Carver B, Castellano D, Chung BH, Clarke $\mathrm{N}$ et al: Management of Patients with Advanced Prostate Cancer: The Report of the Advanced Prostate Cancer Consensus Conference APCCC 2017. Eur Urol 2018, 73(2):178-211.

6. Yousef GM, Obiezu CV, Luo LY, Magklara A, Borgono CA, Kishi T, Memari N, Michael I P, Sidiropoulos $M$, Kurlender $L$ et al: Human tissue kallikreins: from gene structure to function and clinical applications. Advances in clinical chemistry 2005, 39:11-79.

7. Borgono CA, Michael IP, Diamandis EP: Human tissue kallikreins: physiologic roles and applications in cancer. Molecular cancer research : MCR 2004, 2(5):257-280.

8. Adamopoulos PG, Kontos CK, Scorilas A: Discovery of novel transcripts of the human tissue kallikrein (KLK1) and kallikrein-related peptidase 2 (KLK2) in human cancer cells, exploiting NextGeneration Sequencing technology. Genomics 2019, 111(4):642-652.

9. Cravens SL, Stivers JT: Comparative Effects of lons, Molecular Crowding, and Bulk DNA on the Damage Search Mechanisms of hOGG1 and hUNG. Biochemistry 2016, 55(37):5230-5242.

10. Martin NE, Mucci LA, Loda M, Depinho RA: Prognostic determinants in prostate cancer. Cancer J 2011, 17(6):429-437.

11. Buhmeida A, Pyrhönen S, Laato M, Collan Y: Prognostic factors in prostate cancer. Diagn Pathol 2006, 1:4-4.

12. Khorana AA, Connolly GC: Assessing risk of venous thromboembolism in the patient with cancer. $J$ Clin Oncol 2009, 27(29):4839-4847.

13. Alexander M, Kirsa S, Wolfe R, MacManus M, Ball D, Solomon B, Burbury K: Thromboembolism in lung cancer - an area of urgent unmet need. Lung Cancer 2014, 84(3):275-280.

14. Falanga A: Thrombophilia in cancer. Semin Thromb Hemost 2005, 31(1):104-110.

15. Fernandez PM, Patierno SR, Rickles FR: Tissue factor and fibrin in tumor angiogenesis. Semin Thromb Hemost 2004, 30(1):31-44.

16. Marfia G, Ampollini A, Navone SE, Di Vito C, Bornati A, Miozzo M, De Rezende G, Rampini P, Riboni L, Mancuso ME et al: Increased VEGF levels in a case of papillary tumor of the pineal region with intracranial hemorrhage: a potential surrogate indicator of tumor angiogenesis and aggressiveness? J Neurosurg Sci 2020, 64(1):107-112.

17. Lin Y, Liu Z, Qiu Y, Zhang J, Wu H, Liang R, Chen G, Qin G, Li Y, Zou D: Clinical significance of plasma D-dimer and fibrinogen in digestive cancer: A systematic review and meta-analysis. Eur J Surg Oncol 2018, 44(10):1494-1503.

18. Amirkhosravi A, Meyer T, Amaya M, Davila M, Mousa SA, Robson T, Francis JL: The role of tissue factor pathway inhibitor in tumor growth and metastasis. Seminars in thrombosis and hemostasis 2007, 33(7):643-652. 
19. Falanga $A$, Marchetti $M$, Vignoli A, Balducci D: Clotting mechanisms and cancer: implications in thrombus formation and tumor progression. Clinical advances in hematology \& oncology : H\&O 2003, 1(11):673-678.

20. Langer F: [Haemostatic aspects in clinical oncology]. Hamostaseologie 2015, 35(2):152-164; quiz 165.

21. Fibrinogen (Fibrin) Degradation Products/D-dimer. In: Clinical Veterinary Advisor. edn. Edited by Wilson DA. Saint Louis: W.B. Saunders; 2012: 933.

22. Li XH, Wang XP, Gu WS, Lin JH, Huang H, Kang T, Zhang L, Chen H, Zheng X: Clinical Significance of Preoperative Thrombin Time in Patients with Esophageal Squamous Cell Carcinoma following Surgical Resection. PLoS One 2015, 10(10):e0140323.

23. Mischke $\mathrm{R}$, Wolling $\mathrm{H}$ : Influence of fibrinogen degradation products on thrombin time, activated partial thromboplastin time and prothrombin time of canine plasma. Haemostasis 2000, 30(3):123130.

24. Sun M, Shariat SF, Cheng C, Ficarra V, Murai M, Oudard S, Pantuck AJ, Zigeuner R, Karakiewicz PI: Prognostic factors and predictive models in renal cell carcinoma: a contemporary review. Eur Urol 2011, 60(4):644-661.

25. Ost P, Decaestecker K, Lambert B, Fonteyne V, Delrue L, Lumen N, Ameye F, De Meerleer G: Prognostic factors influencing prostate cancer-specific survival in non-castrate patients with metastatic prostate cancer. Prostate 2014, 74(3):297-305.

\section{Tables}

Table 1. The distribution of subgroups in training and validation cohorts. 


\begin{tabular}{|c|c|c|c|c|}
\hline & $\begin{array}{l}\text { Training } \\
(\mathrm{n}=120)\end{array}$ & $\begin{array}{l}\text { Validation } \\
(n=48)\end{array}$ & $\begin{array}{l}\text { Entire } \\
(\mathrm{n}=168)\end{array}$ & $P$ value \\
\hline \multicolumn{5}{|c|}{ Age, years } \\
\hline$\leq 60$ & 61 & 26 & 87 & 0.696 \\
\hline$>60$ & 59 & 22 & 81 & \\
\hline \multicolumn{5}{|c|}{ TPSA, ng/ml } \\
\hline$\leq 10$ & 45 & 14 & 59 & 0.307 \\
\hline$>10$ & 75 & 34 & 109 & \\
\hline \multicolumn{5}{|c|}{ Gleason score } \\
\hline 6 & 26 & 8 & 34 & \\
\hline 7 & 30 & 12 & 42 & \\
\hline 8 & 22 & 10 & 32 & \\
\hline 9 & 20 & 7 & 27 & \\
\hline 10 & 22 & 11 & 33 & \\
\hline \multicolumn{5}{|c|}{ Pathology T } \\
\hline 1 & 15 & 6 & 21 & 0.81359 \\
\hline 2 & 76 & 29 & 105 & \\
\hline 3 & 27 & 11 & 38 & \\
\hline 4 & 2 & 2 & 4 & \\
\hline \multicolumn{5}{|c|}{ FDP, $\mu \mathrm{g} / \mathrm{ml}$} \\
\hline$\leq 1.85$ & 59 & 27 & 86 & 0.407 \\
\hline$>1.85$ & 61 & 21 & 82 & \\
\hline
\end{tabular}

RFS, recurrence-free survival; TT, thrombin time; FDP, fibrin degradation products; TPSA, total prostatespecific antigen.

Table 2. The risk points of each variable in the nomogram. 


\begin{tabular}{|llllll|}
\hline Parameters & \multicolumn{4}{l|}{ Subgroups } \\
\hline Age, years & $>60$ & $\leq 60$ & & & \\
\hline Points & 0 & 28.93 & & & \\
\hline TPSA, $\mathrm{ng} / \mathrm{ml}$ & $\leq 10$ & $>10$ & & & \\
\hline Points & 0 & 14.02 & & & \\
\hline Gleason score subgroup & 1 & 2 & 3 & 4 & 5 \\
\hline Points & 0 & 25 & 50 & 75 & 100 \\
\hline TT, $\mathrm{s}$ & $>17.1$ & $\leq 17.1$ & & & \\
\hline Points & 0 & 68.64 & & & \\
\hline FDP, $\mu \mathrm{g} / \mathrm{ml}$ & $>1.85$ & $\leq 1.85$ & & \\
\hline Points & 0 & 26.09 & & \\
\hline
\end{tabular}

TT, thrombin time; FDP, fibrin degradation products; TPSA, total prostate-specific antigen

Figures 


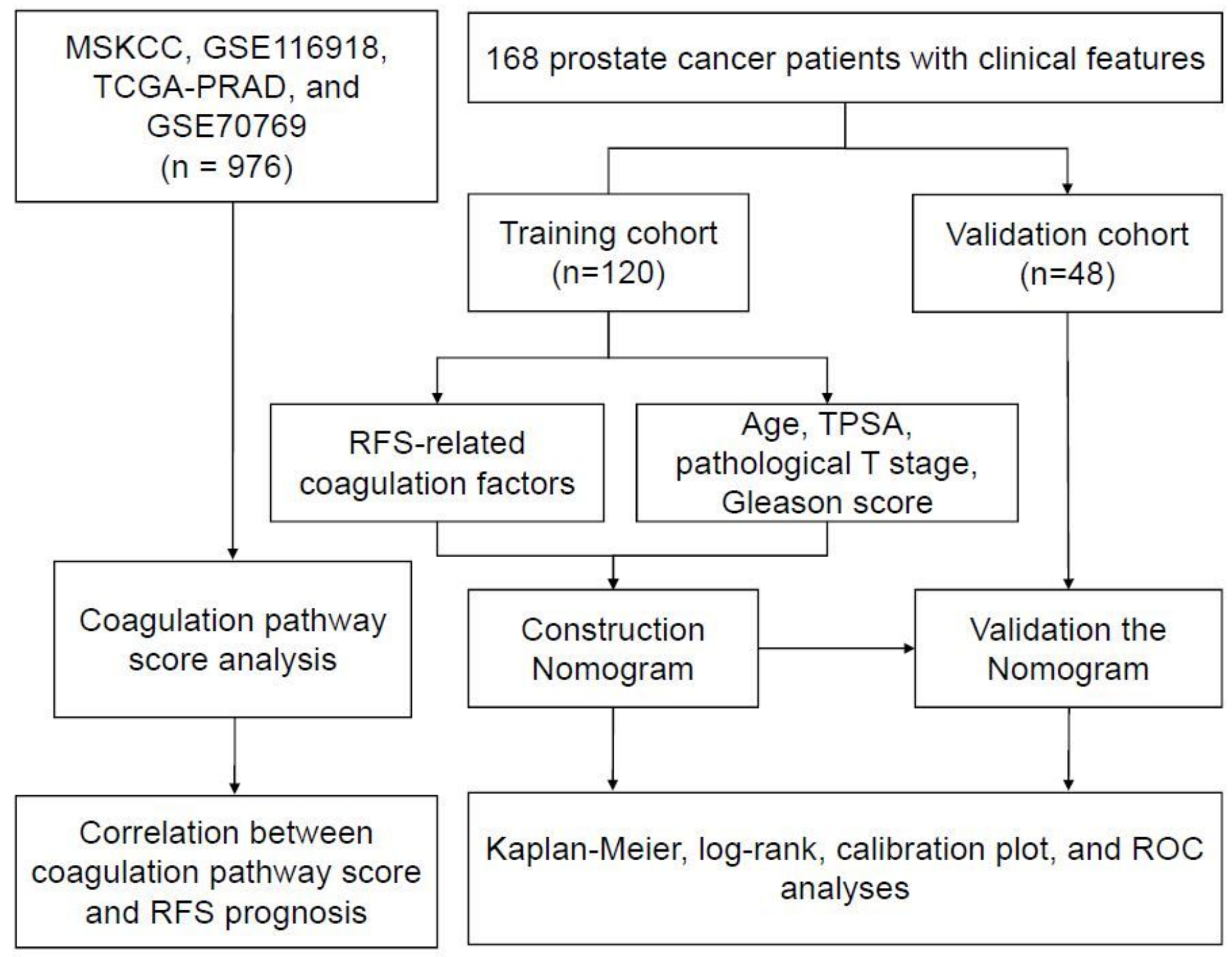

Figure 1

The flow chart showed the design of the current study. 

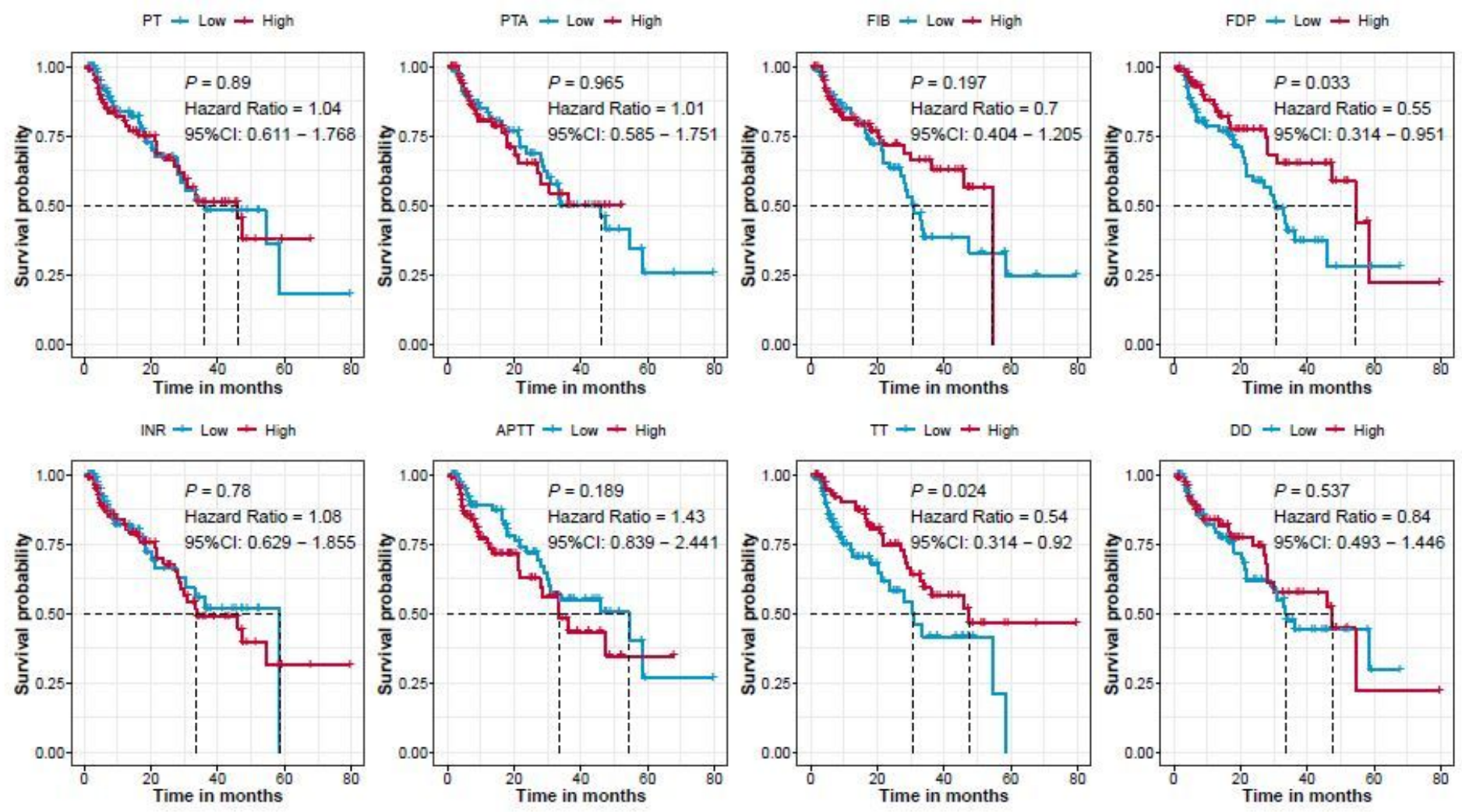

Figure 2

Kaplan-Meier survival analysis of coagulation pathway active status and recurrent-free survival. 
Points

Age, years

TPSA, $\mathrm{ng} / \mathrm{ml}$

Gleason Group

TT, s

FDP

Total Points
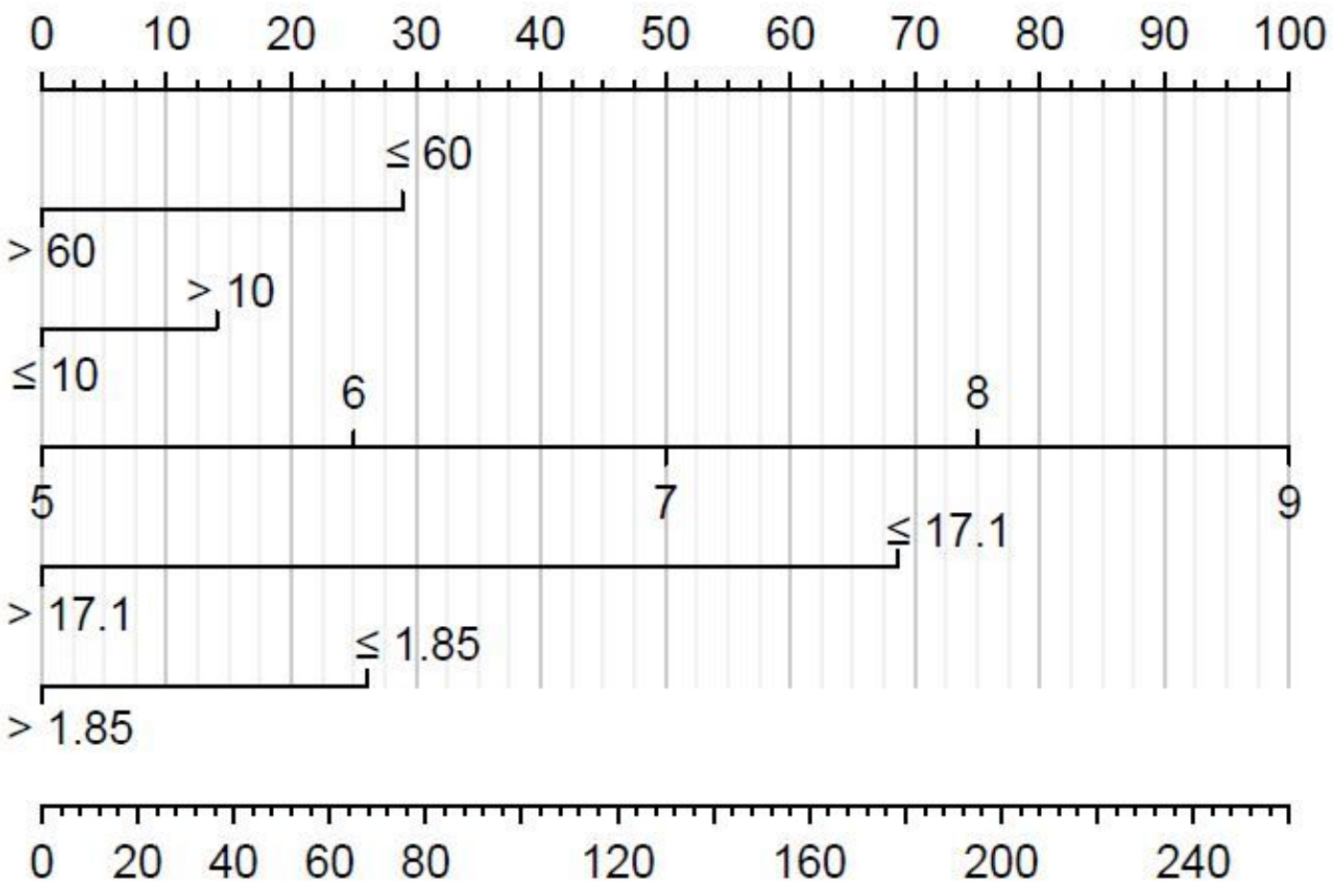

Recurrence Risk

\begin{tabular}{lllllll}
\hline 0.2 & 0.3 & 0.4 & 0.5 & 0.6 & 0.7 & 0.8
\end{tabular}

Figure 3

Construction of a nomogram for recurrence-free survival predicting. Nomogram combining signature with laboratory results \& clinicopathological features. TPSA = total prostate-specific antigen $(\mathrm{ng} / \mathrm{ml}) ; \mathrm{TT}=$ plasma prothrombin time (sec); 1 -year recurrence $=$ recurrence-free survival for 1 year; 3 -year recurrence $=$ recurrence-free survival for 3 years. To obtain the nomogram-predicted probability of recurrent-free survival possibility, get the location of different status at each axis and then draw a vertical line to the "Point" axis to determine the points of each variable value. Sum the points for all value got from the calculation before. Locating the sum on the "Total Points" line to be able to assess the individual probability of recurrent-free survival. 


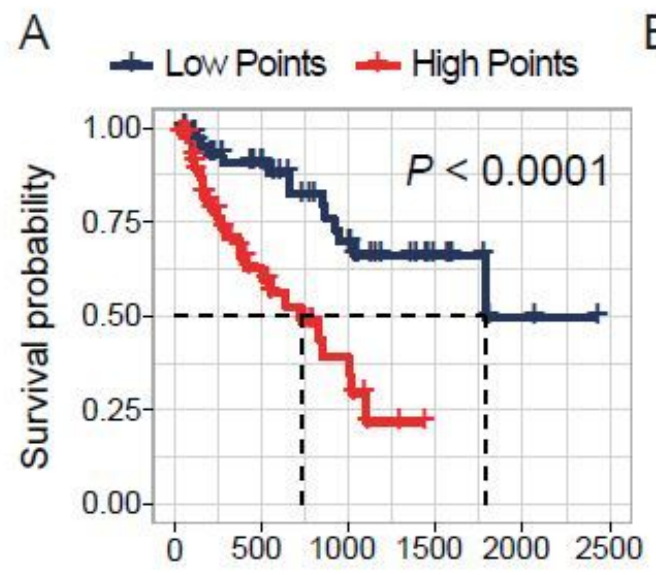

B

Number at risk
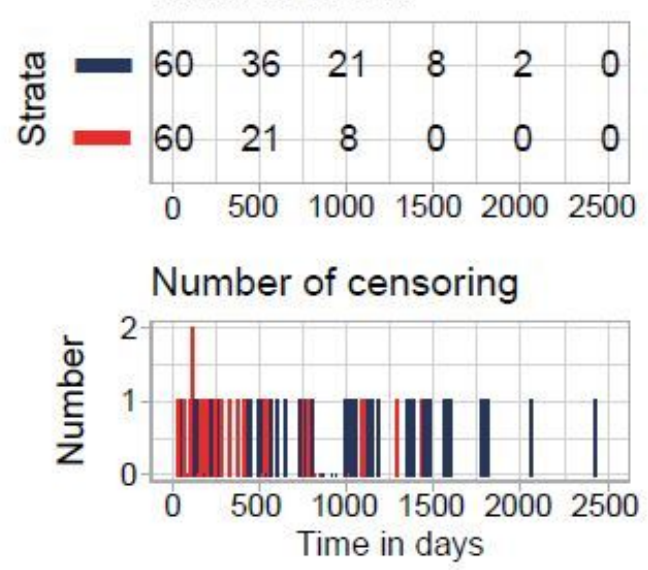
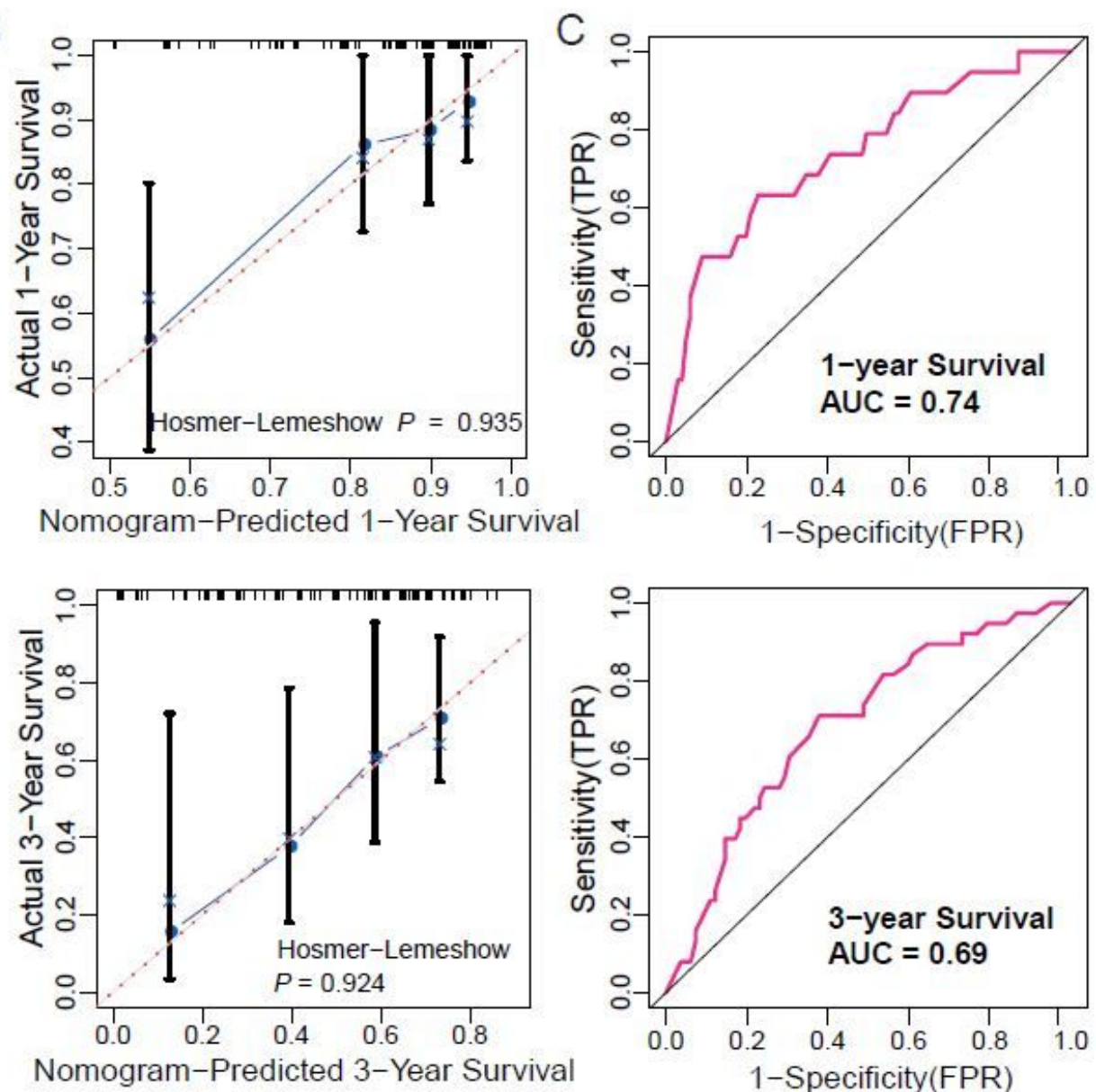

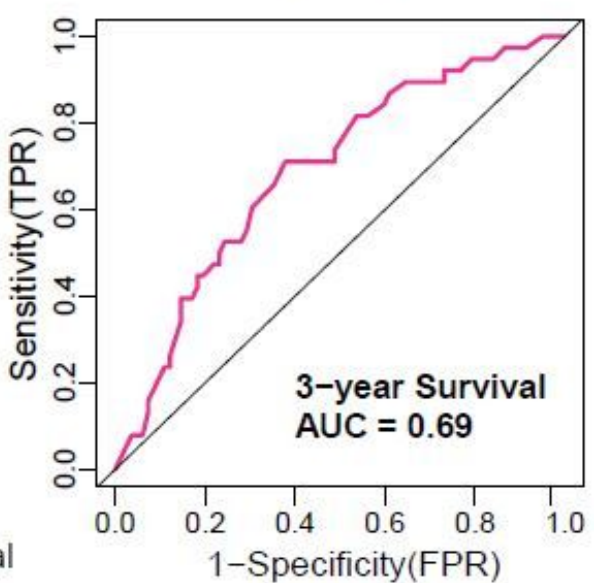

Figure 4

Verifying the accuracy of the Nomogram. A. Stratified survival analyses based on the risk score in the training cohort. B. Calibration plot displaying that the nomogram model predicted recurrence-free survival probabilities was proved consistent with the observed proportions. C. ROC curve analyses for the Nomogram in predicting recurrence-free survival. ROC curves showed satisfied predictive values of the Nomogram in the training cohort at 1- and 3-year. ROC, Receiver Operating Characteristic. 

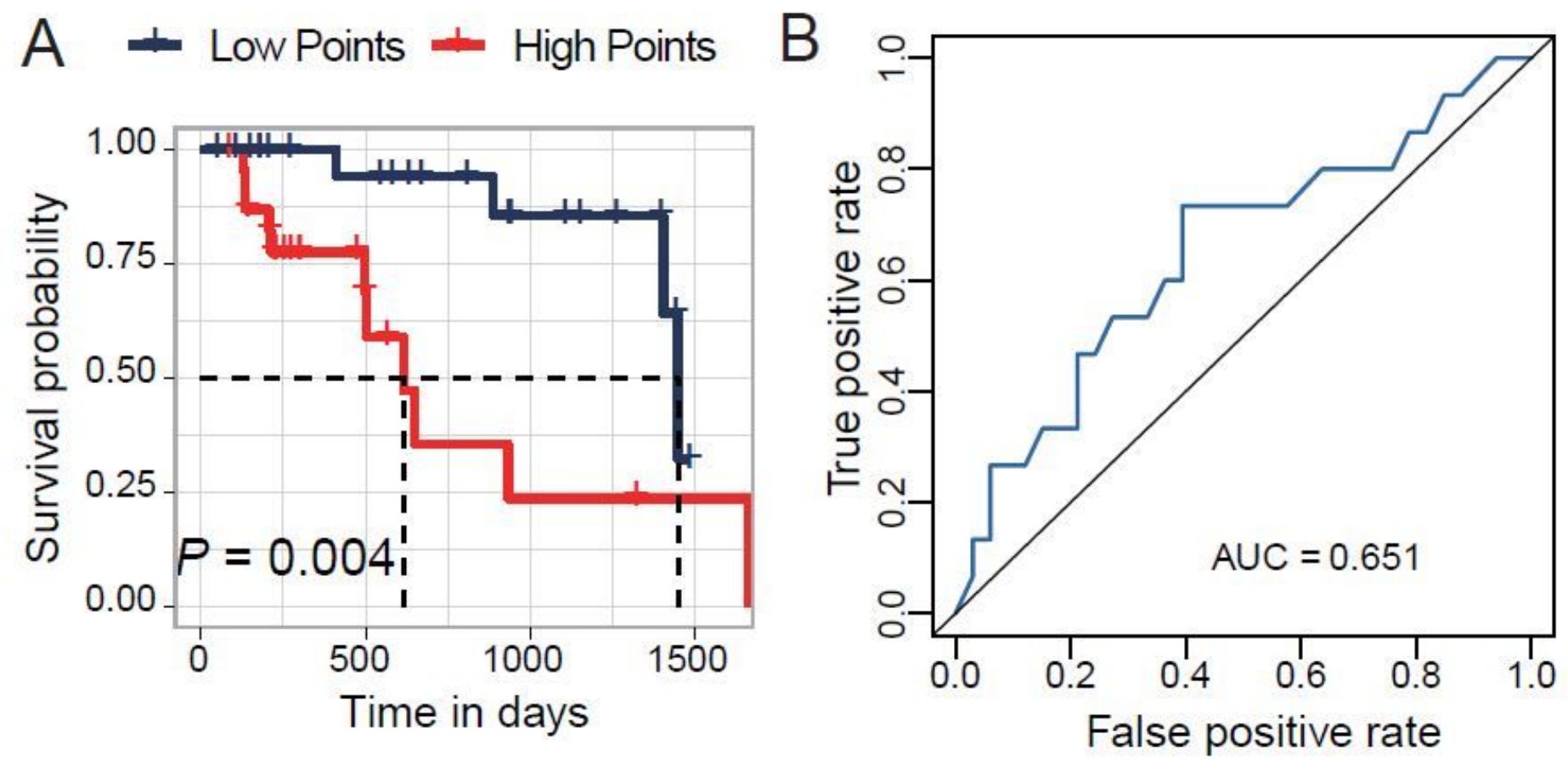

Figure 5

Verifying the accuracy of the Nomogram in the validation cohort. A. Stratified survival analyses based on the risk score in the validation cohort. B. ROC curves showed satisfied predictive values of the Nomogram in the validation cohort at 1-and 3-year. ROC, Receiver Operating Characteristic. 
TPSA $<=10$

Strata $\div$ High-risk $\div$ Risk=low

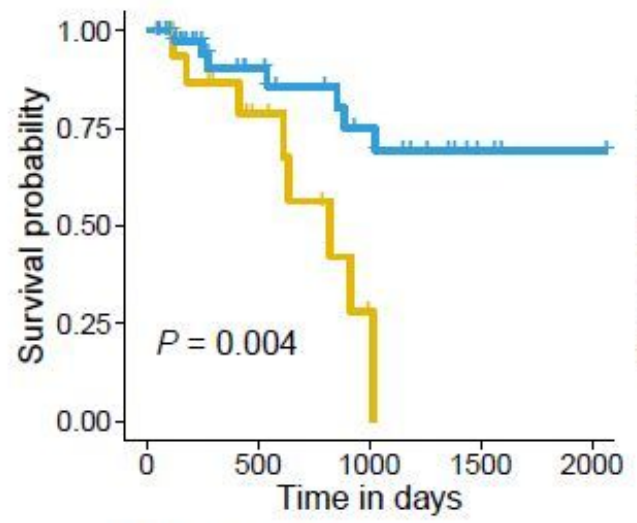

TPSA $>10$

Strata + High-risk $\perp$ Low-risk

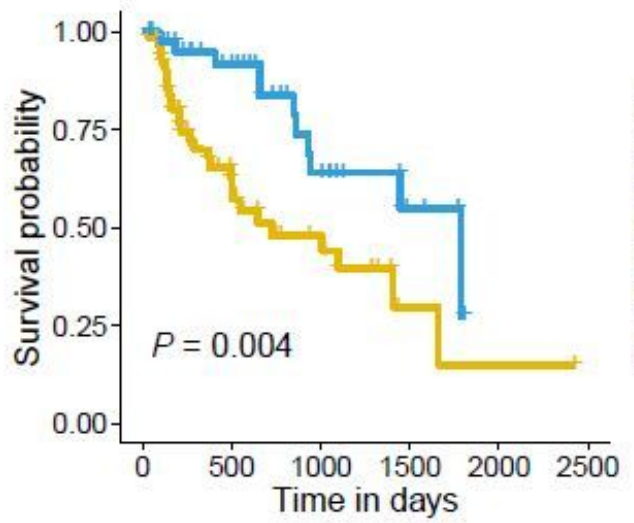

Age $<=60$

Strata $\div$ High-risk $\div$ Low-risk

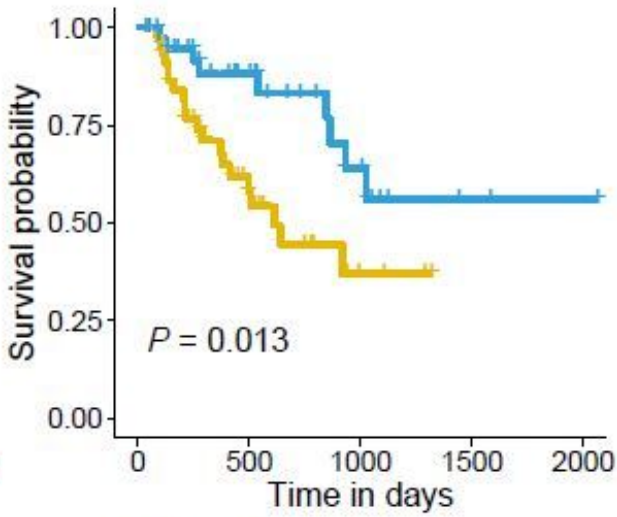

Age $>60$

Strata + High-risk $\perp$ Low-risk

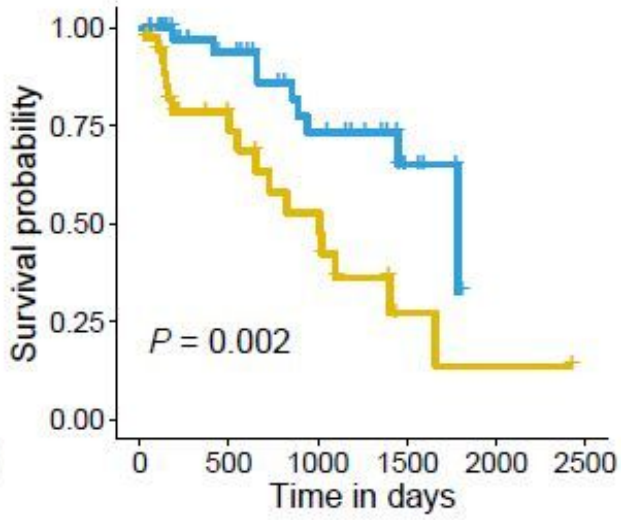

Gleason score $<=7$

Strata $\div$ High-risk $\perp$ Low-risk

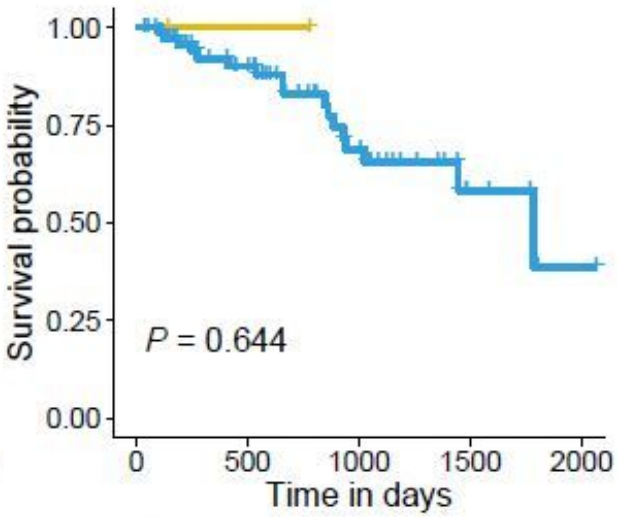

Gleason score $>7$

Strata + High-risk $\neq$ Low-risk

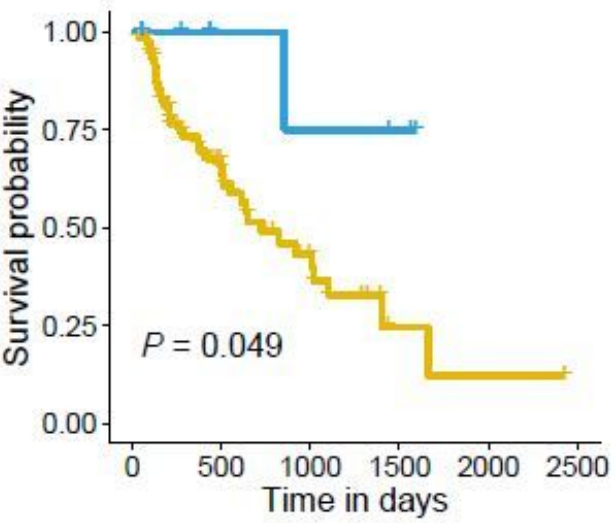

Figure 6

Subgroup Kaplan-Meier analyses according to different clinicopathological features, including age, PSA level, and Gleason score. 
MSKCC Cohort

- High (48) - Low (92)

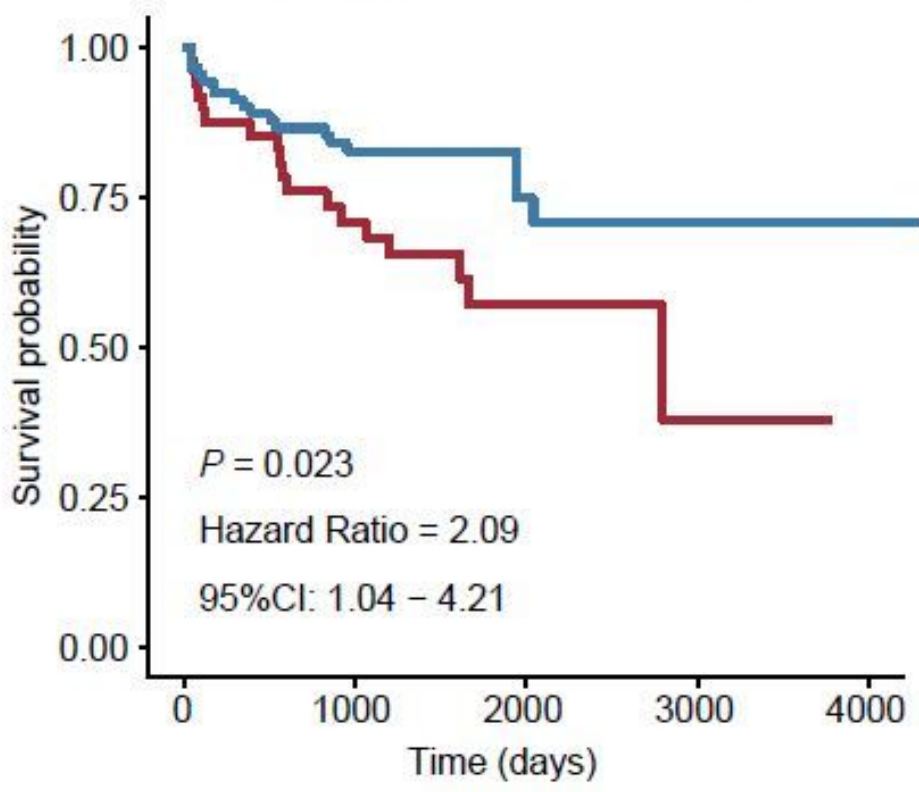

TCGA-PRAD Cohort

- High (340) Low (155)

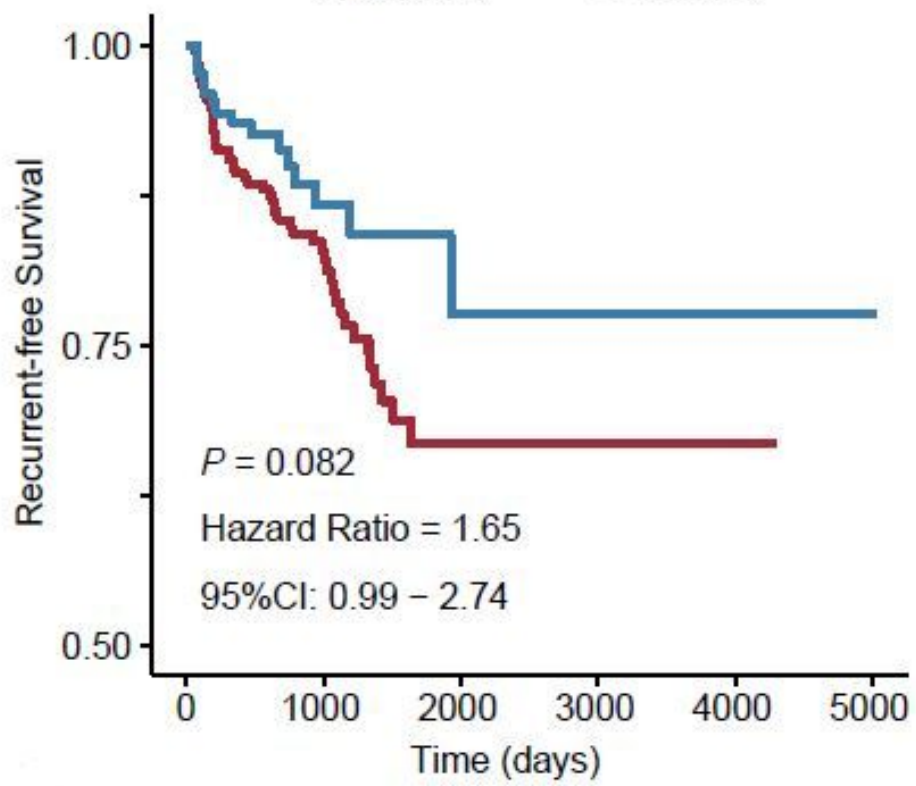

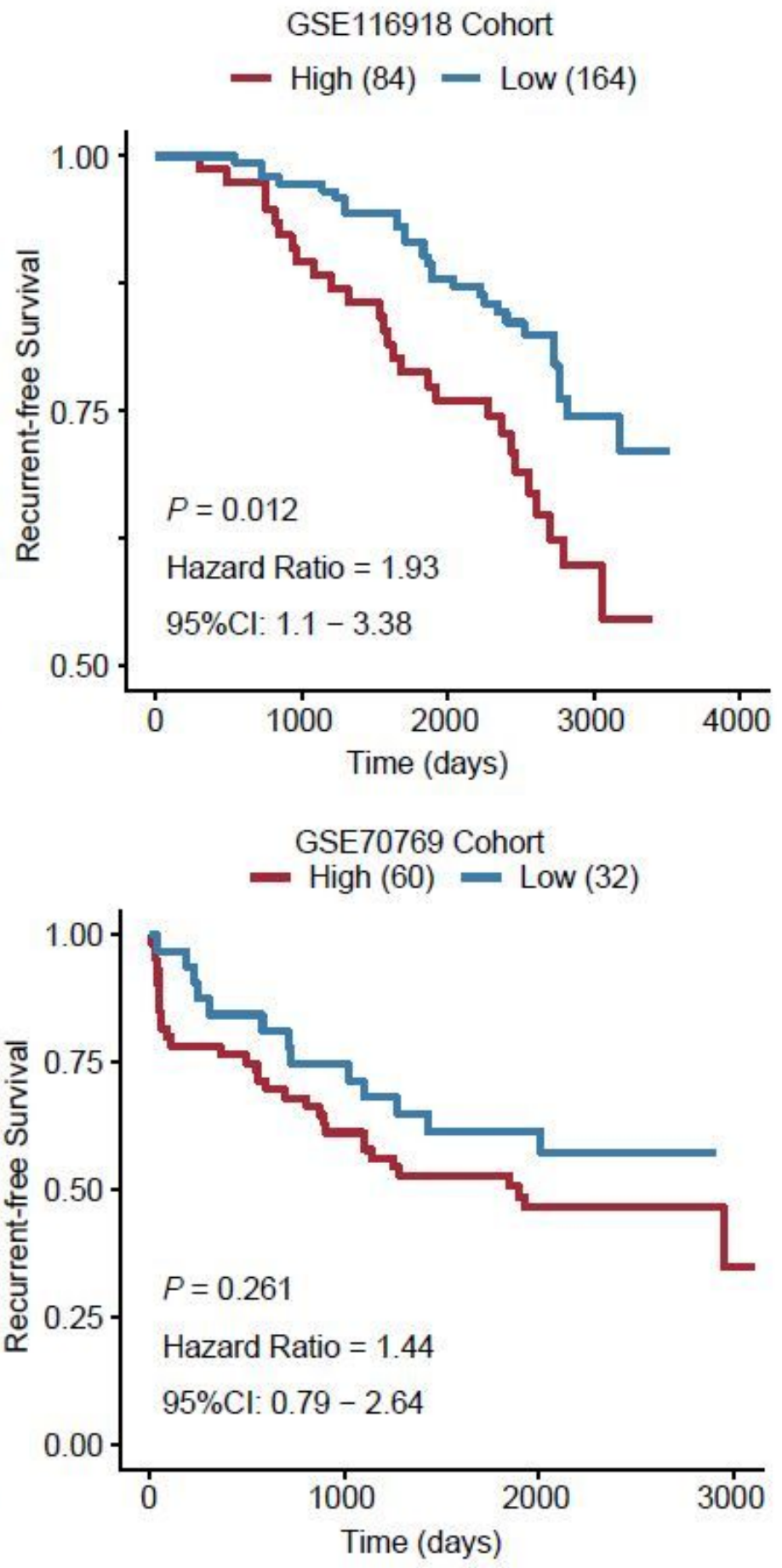

\section{Figure 7}

Kaplan-Meier plot and log-rank analyses to compare the recurrence-free survival difference of patients with high and low coagulation pathway scores determined by gene set variation analysis. 